\title{
Focused Ion Beam investigations on Cement based Materials in aggressive aqueous environments
}

\author{
M. Schwotzer, ${ }^{*}$ T. Scherer, ${ }^{* *}$ Ch. Kübel, ${ }^{* *}$ and A. Gerdes*,*** \\ * Institute of Functional Interfaces, Karlsruhe Institute of Technology, Karlsruhe, Germany \\ ** Institute for Nanotechnology, Karlsruhe Institute of Technology, Karlsruhe, Germany \\ *** Institute for Prevention in Construction, University of Applied Sciences, Karlsruhe, Germany
}

Porous mineral materials such as cement based systems are used in construction and in various technical applications. In practice material failure is not only caused by mechanical load but also by exposure to aggressive environments. These materials often are multi component systems with a pore system characterized by pore sizes ranging from the nanometer up to the millimeter scale. Commonly, the pore system contains an aqueous solution with a complex composition. The chemical stability of these porous mineral systems is generally determined by chemical equilibria between the pore solution and the solid phases. In their multifaceted application fields, cement based materials come in contact with natural waters of various composition. It's inevitable that these equilibria will be disturbed during the use of the materials. Due to concentration gradients between the pore solution of these porous materials and a surrounding aqueous media reactive transport processes are initiated, causing dissolution and crystallization reactions. The materials are subject to chemical changes affecting their durability. Generally many mechanisms of complex damaging reactions, for instance occurring in the presence of sulfate ions are still debatable [1]. In order to investigate the material performance of cementitious systems usually the long term behavior (weeks and months) of these materials has been studied (e.g. [2]). In this context previous studies showed that the properties of the material/water interface are of relevance. Reactive transport processes are able to cause substantial structural and chemical changes in the surface zone of cement based materials [3]. A detailed knowledge of the reaction mechanisms is necessary to develop reliable prognostic models with respect to the durability of materials.

In order to investigate the relevance of chemical and micro structural changes in the material/water interface short term experiments were carried out. Freshly cut surfaces of cement paste samples prepared with white Portland cement were exposed to aqueous solutions with different chemical compositions, such as hard tap water and a $0.1 \mathrm{M} \mathrm{MgSO}_{4}$ solution. The Focused Ion Beam technique enables detailed investigations of surface reactions. Imaging and EDX investigations of the profile cuts reveal structural and chemical properties of the material/water interface. In both experiments a crystalline covering layer developed on the sample surface (Fig. 1). The sample exposed to hard tap water showed a dense covering layer of $\mathrm{CaCO}_{3}$ crystals (Fig. 1 a) whereas the sample exposed to the $\mathrm{MgSO}_{4}$ solution was covered by flaky $\mathrm{Mg}(\mathrm{OH})_{2}$ crystals (Fig. 1 b). Both types of crystalline coverings are considered to provide a protective effect ([4] and [5]).

EDX measurements indicate that the dense $\mathrm{CaCO}_{3}$ coating provides a protective effect against the leaching of $\mathrm{Ca}^{2+}$ (Fig. 2 a). In contrast to that the $\mathrm{Mg}(\mathrm{OH})_{2}$ layer seemed not to constrain transport processes as mentioned e.g. in [5]. The continuous progress of transport processes was indicated by EDX investigations, as a significant decrease of $\mathrm{Ca}^{2+}$ and ingress of $\mathrm{Mg}^{2+}$ in the surface near $\mu \mathrm{m}$ was observed. This was accompanied by a considerable ingress of sulfate ions into deeper areas (Fig. 1b).

The results presented here illustrate, that the chemical and structural properties of the material/water interface have a consequential influence on reactive transport processes. In particular the 
compactness of crystalline covering layers, related to crystallization mechanisms, is of relevance. These properties can change already in a time scale of hours due to reactive transport caused by differences between the hydrochemical properties of a surrounding aqueous solution and the pore solution of the material. Besides the essential long term investigations, the deduction of detailed reaction models can be complemented by studies of the reactivity of the material/water interface with a special focus on the processes in the surface near $\mu \mathrm{m}$. In order to investigate the reactions on a smaller scale in the material/water interface as well as in the cement-paste/reaction-productinterface, TEM investigations of the interaction zone (e.g. dotted line in Fig. 1a) were performed and related EDX- and EELS-spectra were measured. Small scale investigation of chemical interactions in these interfaces will help to improve the durability of high performance cement based materials.

\section{References}

[1] A. Neville, Cem. Concr. Res. 34 (2004) 1275-1296

[2] Y. Maltais et al., Cem. Concr. Res. 34 (2004) 1579-1589

[3] M. Schwotzer, PhD thesis, Universität Karlsruhe (2008) 189

[4] E. Koelliker., 8th Int. Congr. Chem. Cement, Rio de Janeiro (1986) 155-164

[5] M. Santhanam et al., Cem. Concr. Res. 33 (2003) 341-346
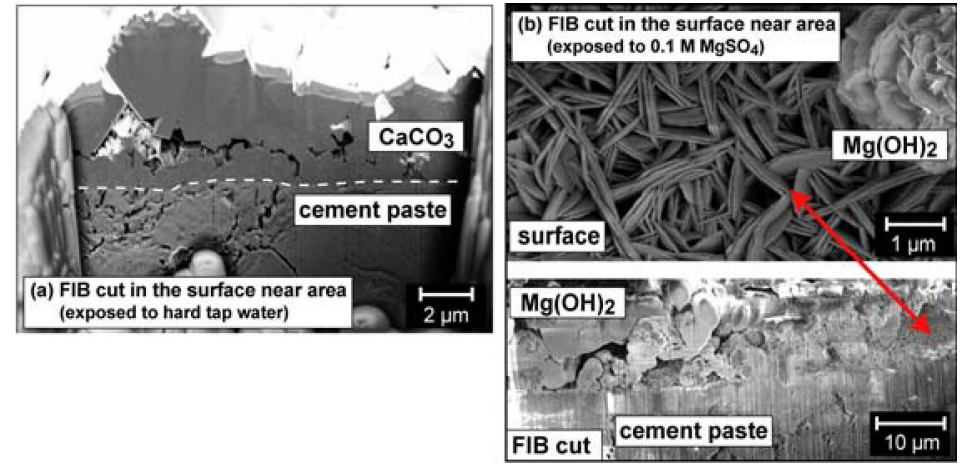

FIG. 1. FIB cuts of a sample exposed to hard tap water (a) an of a sample exposed for $4 \mathrm{~h}$ to a $\mathrm{MgSO}_{4}$ solution showing the sample surface and a FIB cross section (b).
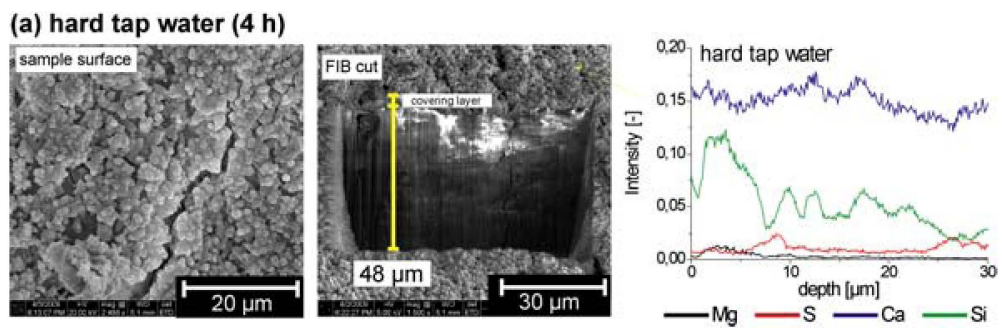

(b) $0,1 \mathrm{M} \mathrm{MgSO}_{4}(4 \mathrm{~h})$
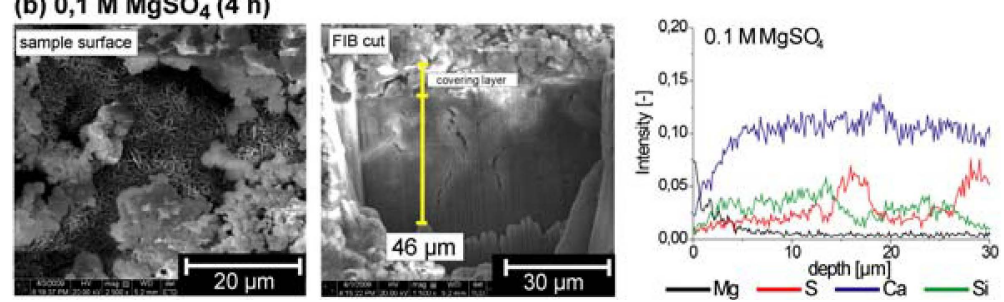

FIG. 2. FIB cuts in the cement paste/water interface of a sample exposed for $4 \mathrm{~h}$ to (a) hard tap water and to (b) $\mathrm{MgSO}_{4}$ solution; right: EDX measurements along the FIB cuts (yellow line) 